\title{
Endoscopic-assisted probing for congenital nasolacrimal duct obstruction
}

\begin{abstract}
Purpose To determine the success rate of initial probing in children with congenital nasolacrimal duct obstruction (CNLDO) at different ages using nasal endoscopy, and to identify the anatomical sites of blockage within the drainage system.

Methods A total of 87 eyes from 67
\end{abstract} consecutive children with CNLDO underwent endoscopic nasolacrimal duct (NLD) probing under general anaesthetic. Patients who had had previous probings were excluded from the study. Diagnosis was based on history of epiphora since birth or shortly after, and fluorescein dye disappearance test (FDDT). Cure was judged as complete or near-complete remission of symptoms and signs and a normal FDDT.

Results The age range was 12-91 months (mean 32.3 months). The success rates of probing were: $100 \%(58 / 58)$ for atresia and stenosis at the lower NLD, $100 \%(13 / 13)$ for punctal stenosis, $55.6 \%(5 / 9)$ for functional epiphora, and $0 \%(0 / 7)$ for canalicular and upper NLD obstruction. Age was not found to significantly affect the outcome of probing, whereas site did. Obstruction at canalicular or upper NLD level became more common with increasing age.

Conclusion Probing of the nasolacrimal system using the endoscopic approach allows direct visualisation of the distal nasolacrimal duct, which facilitates diagnosis of the anomaly at this site. More complex proximal anomalies became increasingly prevalent in older children, which accounted for the poorer results with increasing age. Site of obstruction has a greater bearing on outcome than patient age.

Eye (2006) 20, 998-1003. doi:10.1038/sj.eye.6702049; published online 2 September 2005
EJ Wallace ${ }^{1}$, A Cox ${ }^{1}$, P White ${ }^{2}$ and CJ MacEwen ${ }^{1}$

Keywords: probing; congenital nasolacrimal duct obstruction; nasal endoscopy; success rate; site of obstruction

\section{Introduction}

Epiphora in infancy is commonly caused by congenital nasolacrimal duct obstruction (CNLDO). This is typically a mucous membrane obstruction at the lower end of the nasolacrimal duct, estimated to be present in up to $20 \%$ of newborn infants. ${ }^{1-5}$ Usually, this is of no clinical significance as the vast majority of affected children will have spontaneous canalisation of the duct and resolution of symptoms before the age of 1 year. ${ }^{3,6,7-10}$

Probing of the lacrimal system is the standard treatment for those children who remain symptomatic of epiphora. However, the timing of probing is a subject of debate, as some reports claim high success rates with early probing (before 12 months), ${ }^{4,11,12}$ although there is no controlled study of probing during the first year of life. Other reports have suggested that the success rate of probing is dictated not by the age at probing, but by the type of obstruction. ${ }^{13-17}$

Simple obstructions at the valve of Hasner are the most common, but more complex anomalies do exist, which may be less amenable to disruption by simple probing alone.

Endoscopic-assisted probing facilitates visualisation of the probe in the membranous nasolacrimal duct and in the inferior meatus of the nose. This may more precisely identify the site of obstruction and provide predictive information regarding treatment options.

The aims of this study were:

(1) to identify the success rate of initial probing in children with CNLDO at different ages;

(2) to locate the anatomical sites of obstruction within the lacrimal drainage system; and 
(3) to determine whether age at probing or site of obstruction has any prognostic significance for the outcome of probing.

\section{Methods}

In a prospective, interventional case series, 87 lacrimal systems from 67 consecutive children with congenital nasolacrimal duct obstruction underwent endoscopic nasolacrimal duct probing between 1996 and 2003. All surgery was carried out under light general anaesthesia by an ophthalmologist and otolaryngologist, the latter experienced in endoscopic sinus surgery.

A diagnosis of presumed CNLDO was made based on a history of epiphora within the first few weeks of life, and an abnormal fluorescein dye disappearance test (FDDT) on examination. ${ }^{18}$ Children who had had previous probings and those who had other causes of epiphora, for example, eyelid malposition, were excluded from the study.

The technique of probing and irrigation has been described previously, ${ }^{19,20}$ but to reiterate: five drops of xylometazoline hydrochloride $0.05 \%$ (paediatric Otrovine) were placed in the nasal cavity immediately after induction of anaesthesia. This was followed by the precise placement of two neurosurgical pledgets soaked in paediatric Otrovine-one pledget under the inferior turbinate, the other between the inferior turbinate and nasal septum in order to constrict the vascular nasal mucosa and improve visualisation. Thereafter, the pledgets were removed and a careful intranasal examination performed. This was done in order to identify any pre-existing nasal pathology, for example, an impacted inferior turbinate. The lacrimal punctae were inspected and punctal stenosis, if present, was treated by dilatation \pm a 3 snip procedure.

The patency of the system was tested by irrigation with dilute fluorescein dye. A cannula was introduced via the upper canaliculus as far as the lacrimal sac and dye injected through the system from a syringe. Nasal endoscopy and irrigation were performed simultaneously and free flow of fluorescein from the lacrimal sac to the nose confirmed anatomical patency of the system.

If no anatomical obstruction was present, and dye was seen to enter the nose in a smooth, steady flow, the child was diagnosed with 'functional' epiphora, that is, tearing secondary to physiological dysfunction (Figure 1).

If no dye was seen passing into the inferior meatus or if fluorescein had to be injected through the system by force (Figure 2), inferior turbinate infracture was performed. This not only improved visualisation of the distal end of the nasolacrimal duct, but was also thought to establish patency in some cases by stretching open the mucosal

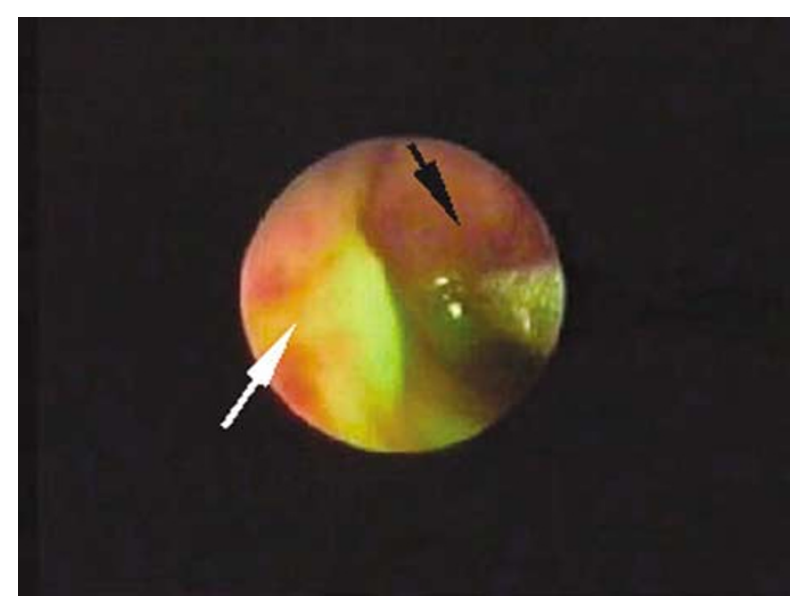

Figure 1 Normal flow of fluorescein through Hasner's valve at the distal nasolacrimal duct (right nostril). White arrowhead denotes Hasner's valve, black arrowhead denotes inferior turbinate being pushed aside by Freer elevator.

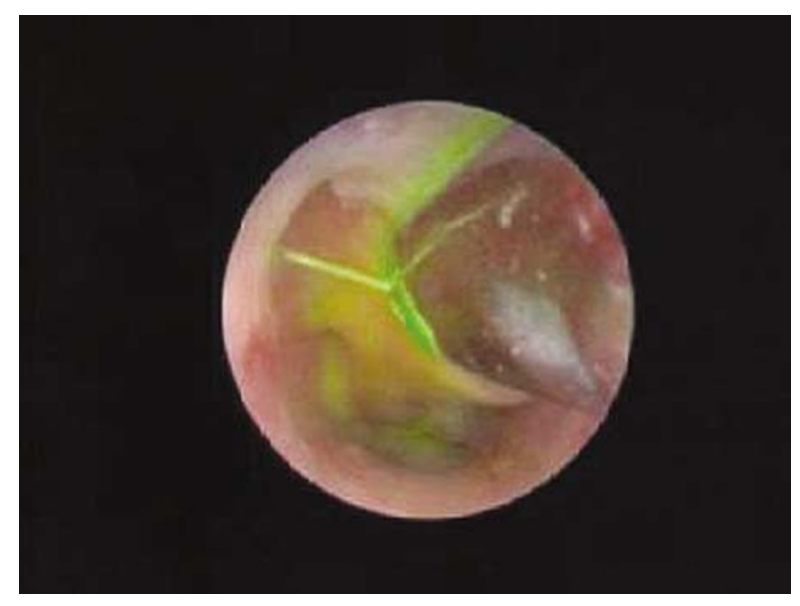

Figure 2 Jet of fluorescein emerging from stenosed Hasner's valve under high pressure (right nostril).

exit of the duct. If repeat irrigation now demonstrated free flow of dye, no further action was taken as the infracture was considered curative.

If, after infracture, fluorescein passed into the inferior meatus in an intermittent or slow manner, or if force was still required to inject dye through the system, a diagnosis of nasolacrimal duct stenosis was made. Failure of dye to appear in the nose, usually associated with ballooning of the nasal mucosa, indicated complete ductal atresia. In cases of stenosis and atresia, probing was performed. The lacrimal probe was passed through the upper punctum and canaliculus, into the sac and onwards into the duct. The probe was observed endoscopically as it entered the inferior meatus. In atretic cases (Figure 3), the mucosa was perforated with a sickle knife if it was not possible to penetrate it using a probe 


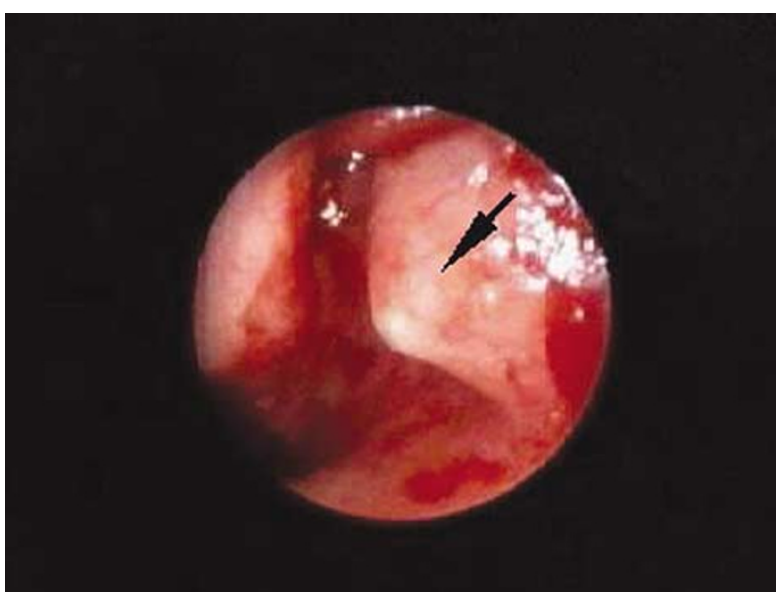

Figure 3 Blanching and tenting of the nasal mucosa due to probe pushing against an atretic valve of Hasner (left nostril). Arrowhead denotes Hasner's valve.

alone. Re-establishment of patency was confirmed by repeating the fluorescein injection.

Children were re-examined 4 weeks postoperatively. If the tearing and discharge were completely cleared and the child had a normal FDDT, this was judged a success. Partial resolution was diagnosed if there was complete or near-complete resolution of epiphora with a persistent relative delay of fluorescein clearance. Failure was defined as a lack of improvement in symptoms or signs.

Data regarding the success rates and sites of obstruction were collected. Binary logistic regression was performed to determine which factors were predictive of success. Since the site of obstruction in patients with stenosis and atresia of the distal nasolacrimal duct was the same, these groups were considered as one. The Wald statistic, used in logistic regression to calculate the significance of the individual regression coefficients, inflated the standard error for large logit coefficients (large effect sizes) and hence caused a type II error. ${ }^{21}$ Therefore, the change in likelihood ratio with the removal of each term was determined and compared with $\chi^{2}$ distribution to determine the effect size for the variables. This is the quoted significant probability or $P$-value. Due to the inflated standard error, the confidence intervals for the regression slope, and hence the odds ratio, are unreliable; so the odds ratio is not quoted.

\section{Results}

A total of 67 children were included in the study and 87 lacrimal drainage systems underwent endoscopic probing. The median age was 29 months $(\mathrm{SD} \pm 18.4)$, with an age range of 12-91 months. In all, 20 cases were bilateral.
For the entire group, probing resulted in a successful resolution of symptoms in $89 \%$. The success rate according to the various age groups is shown in Table 1.

The anatomical sites of blockage within the drainage system were identified for each age group and are summarised in Graph 1. Overall 61 children were diagnosed with nasolacrimal duct obstruction - 39 children had lower nasolacrimal stenosis, 19 had nasolacrimal atresia and three had upper nasolacrimal duct (NLD) obstruction. All three probings that failed in the nasolacrimal group had upper NLD obstruction (see Table 2). Probing was successful in all cases of atresia and stenosis at the distal end of the NLD, although in seven cases the probe passed into the submucosal space and had to be guided into the correct anatomical pathway with the aid of the endoscope.

Table 1 Success rate of probing according to age group

\begin{tabular}{lcr}
\hline Age (months) & Number & Success rate \\
\hline $12-23$ & 29 & $28 / 29(96.4 \%)$ \\
$24-35$ & 28 & $25 / 28(89.3 \%)$ \\
$36-47$ & 15 & $14 / 16(87.5 \%)$ \\
$48-59$ & 7 & $4 / 7(57.1 \%)$ \\
60 or older & 7 & $6 / 7(85.7 \%)$ \\
\hline
\end{tabular}

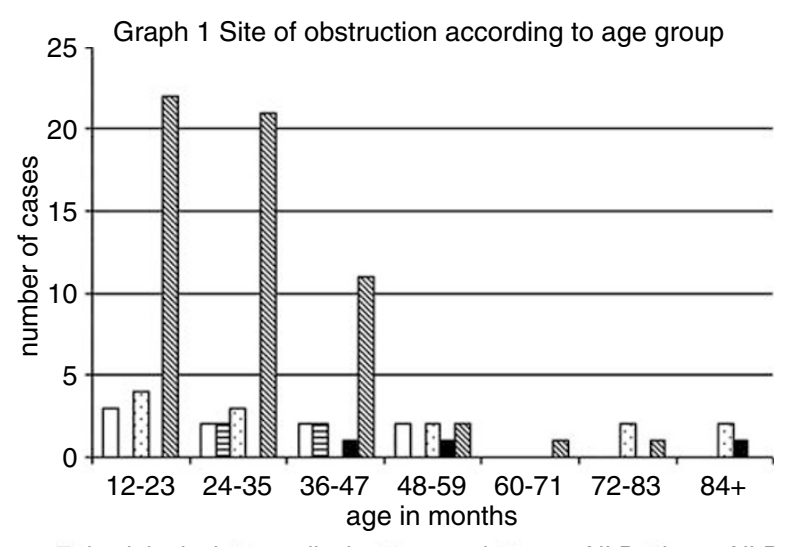

口physiological $\boxminus$ canalicular øpunctal mupper NLD \&lower NLD

Table 2 Success rate of probing according to site of obstruction

\begin{tabular}{lrcc}
\hline $\begin{array}{l}\text { Site of } \\
\text { obstruction }\end{array}$ & Number & $\begin{array}{c}\text { Median age } \\
\text { (months) } \\
\text { (lower-upper quartile) }\end{array}$ & Success rate \\
\hline Functional & 9 & $34(17-46)$ & $5 / 9(55.6 \%)$ \\
Punctal & 13 & $35(13-78)$ & $13 / 13(100 \%)$ \\
Canalicular & 4 & $31(26-37.5)$ & $0 / 4(0 \%)$ \\
Upper NLD & 3 & $52(39-85)$ & $0 / 3(0 \%)$ \\
Lower NLD & 58 & $26(18-36)$ & $58 / 58(100 \%)$ \\
\hline
\end{tabular}




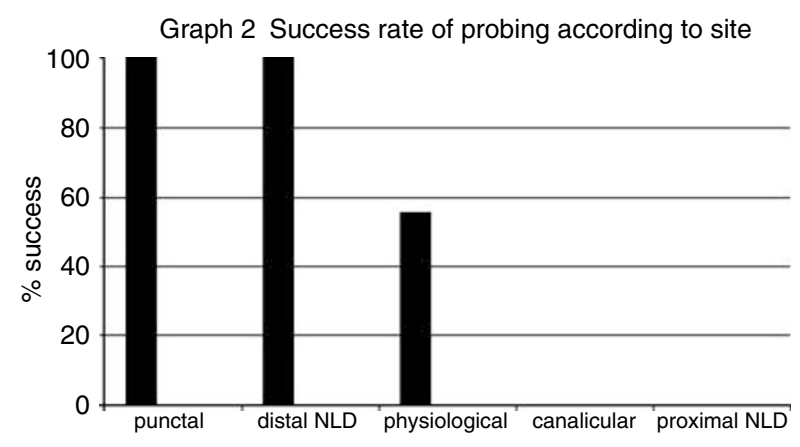

Proximal obstruction in the form of a punctal membrane or stenosis was found to be the cause of symptoms in 13 children, all of whom underwent a successful probing after treatment of the punctal anomaly. Proximal obstruction in the form of canalicular stenosis was found in four patients. In all cases, probing was unsuccessful.

Nine patients had no evidence of anatomical obstruction and five of these had resolution of symptoms after probing, giving a success rate of $55.6 \%$ for this subgroup. The success rate of probing according to the site of obstruction is illustrated in Graph 2.

Atresia and stenosis of the lower NLD accounted for the vast majority of obstructions $(70 \%)$ in this group of children. Physiological blockage and more proximal obstructions of the nasolacrimal system became increasingly prevalent in older children.

Using binary logistic regression, age was not found to significantly affect the outcome of probing $(P=0.83)$, whereas site of obstruction did significantly affect the outcome $(P<0.001)$. Physiological obstruction, canalicular, and upper NLD obstruction were all highly predictive of probing failure, and children with such anomalies were significantly less likely to have a successful probing compared to those with either punctal or lower NLD obstruction $(P<0.001)$. This is summarised in Table 3.

Table 3 Factors predictive of probing failure

\begin{tabular}{lrc}
\hline & $\begin{array}{l}\text { Likelihood } \\
\text { ratio (d.f.) }\end{array}$ & Significance \\
\hline Age & $0.044(1)$ & $P=0.83$ \\
Site of obstruction & $43.45(5)$ & $P<0.001$ \\
$\quad$ Physiological vs distal NLD & $11.96(1)$ & $P<0.001$ \\
Punctal obstruction $v$ distal & $1.20(1)$ & $P=0.27$ \\
NLD & & \\
Canalicular obstruction vs & $13.35(1)$ & $P<0.001$ \\
distal NLD & & \\
$\quad$ Upper NLD obstruction vs & $23.49(1)$ & $P<0.001$ \\
distal NLD & & \\
\hline
\end{tabular}

\section{Discussion}

Epiphora is common in infants and is most frequently due to a congenital nasolacrimal duct obstruction. ${ }^{1,2}$ This is an obstructive membrane at Hasner's valve, which opens spontaneously in approximately $95 \%$ of cases by 1 year of age..$^{3,6,7-10}$

Surgical treatment for CNLDO is probing of the nasolacrimal duct. Timing of this intervention has long been the subject of discussion. Those in favour of early probing (usually before 12 months of age) argue that delays in opening the obstructed system will prolong any inflammation and result in secondary fibrosis, which may reduce the chance of any surgical intervention succeeding. ${ }^{4,11,12}$ Those in favour of late intervention (usually after 12 months of age) 3,7-10,22,23 $^{2}$ argue that the vast majority of children with epiphora will improve spontaneously by 1 year of age, so most children can avoid unnecessary probing which may, in itself, lead to iatrogenic stenosis of the lacrimal passages.

The reported success rates of probing are variable and are often presented as a function of the age of the child at probing, with an inverse relationship between age and success of probing being found. ${ }^{4,12,16,24}$ However, as the natural history of spontaneous resolution is well recognised, , $^{3,7-10,13}$ it has been suggested that the decreasing success rate with increasing age is actually due to the process of natural selection, with cases of simple membranous obstruction at the distal NLD resolving, while more complex obstructions persist and accumulate in number. ${ }^{25}$

This argument has been supported by a controlled study of probing vs observation during the second year of life, which identified that both are equally effective during this time (probing and observation resulted in the same outcome in children with epiphora between 1 and 2 years of age), but that the rate of spontaneous resolution/ probing success was lower than during the first year of life. $^{26}$

We found an overall cure rate of $89 \%$ with the initial attempt at probing. The reason for our higher success rate in comparison to previous studies may have been attributable to the use of nasal endoscopy. This allowed accurate guiding of the probe under direct visualisation in most cases, and therefore a reduction in the number of false passages formed. It also provided a more accurate diagnosis regarding the level and degree of outflow obstruction. Previous studies have been unable to comment on the site of obstruction, having conducted 'blind' probings. This may explain why the success rates have been so variable between studies. Some series may have had a higher rate of undetected false passage formation and hence lower success rates, others having 
had more straightforward obstructions and therefore higher success rates.

The advantages of endoscopy are well recognised. ${ }^{19,20,27-29}$ This technique allows better planning of the probing procedure with the option to carry out additional treatments, if required, under a single anaesthetic. In our study, treatments in the form of inferior turbinate infracture and 'cut down' were facilitated by the use of endoscopy, although children who had such treatments were not matched to any controls.

The group with the highest success rate comprised those children with simple CNLDO, that is, a membranous obstruction at the distal end of the nasolacrimal duct (either stenosis or atresia). All 58 children in this group had a successful probing. The lowest success rates for probing were found in those children with proximal obstruction in the form of canalicular or upper nasolacrimal blockage, and those with physiological obstruction. The three children who had upper nasolacrimal obstruction were cured only after an endonasal dacrocystorhinostomy was carried out. All four patients who had canalicular stenosis required the insertion of silicone tubes to effect a cure.

Proximal obstruction in the form of a punctal membrane or stenosis carried a good prognosis. Once treated with punctal dilatation and/or a 3 snip procedure, this allowed successful passage of a probe and resolution of symptoms in all cases. It is important to note that there were five cases of punctal stenosis/atresia which were also associated with distal outflow obstructions, and therefore all cases with a punctal anomaly should proceed to a full probing.

Nine patients had anatomically patent lacrimal systems. These children, who had symptomatic epiphora and a delayed FDDT, clearly had a functionally inadequate drainage system and were diagnosed with physiological obstruction. This may have been due to a number of factors - inadequate pumping mechanism, nasal mucosal oedema (if present with colds/allergy), or a tight inferior meatus. Five children in this group were 'cured' after probing. This may have been due to infracture of the inferior turbinate, which has been shown to improve cure rates of probing due to stretching of the inferior meatus. ${ }^{30,31}$ Children who remain symptomatic after probing are likely to decline in number with time, possibly due to widening of the face, nose, and lacrimal passages as a result of facial growth. ${ }^{32}$

Our study suggests that age itself is not the primary factor, but rather it is the nature of the obstruction which alters with age that determines the success of probing. This is in keeping with previous work that has suggested that the apparent reduction in probing success with increasing age is due to an accumulation of more complex obstructions as less severe ones spontaneously resolve. $^{25}$

We have demonstrated that accurate localisation of the site of obstruction, which has only been possible with the assistance of nasal endoscopy, can be of prognostic value for children with CNLDO undergoing probing. Distal nasolacrimal duct obstruction, which carries a high success rate with probing, is more common in younger children, while proximal and physiological obstruction, which carry a poorer success rate with probing, represent an increasingly large proportion of the older population.

Therefore, the success rate of probing does tend to reduce with increasing age, but data analysis suggests that it is site and not age that is predictive of probing failure.

These findings suggest that (1) delaying probing is not harmful and (2) probing failure in older children is due to more complex disease that is more difficult to treat successfully with simple probing.

\section{References}

1 Cassady JV. Developmental anatomy of the naso-lacrimal duct. Arch Ophthalmol 1952; 47: 141-158.

2 Schwartz M. Congenital atresia of the naso-lacrimal canal. Arch Ophthalmol 1935; 13: 301-302.

3 Macewen CJ, Young JDH. Epiphora in the first year of life. Eye 1991; 5: 596-600.

4 Ffookes OO. Dacryocystitis in infancy. Br J Ophthalmol 1962; 46: 422-434.

5 Guerry D, Kendig FI. Congenital impotency of the naso-lacrimal duct. Arch Ophthalmol 1948; 39: 193-204.

6 Cassady JV. Dacryocystitis in infancy. Am J Ophthalmol 1948; 31: 773-780.

7 Noda S, Hayasaka S, Tomoichi S. Congenital nasolacrimal duct obstruction in Japanese infants: it's incidence and treatment with massage. J Paediatr Ophthalmol Strabismus 1991; 28: 20-22.

8 Paul TO. Medical management of congenital nasolacrimal duct obstruction. J Paediatr Ophthalmol Strabismus 1985; 22: 68-70.

9 Peterson RA, Robb RM. The course of congenital obstruction of the nasolacrimal duct. J Paediatr Ophthalmol Strabismus 1978; 15: 246-250.

10 Nelson LB, Calhoun JH, Menduke H. Medical management of congenital nasolacrimal duct obstruction. Ophthalmology 1985; 92: 1187-1190.

11 Baker JD. Treatment of congenital nasolacrimal duct obstruction. J Paediatr Ophthalmol Strabismus 1985; 22: 34-35.

12 Katowitz JA, Welsh MG. Timing of initial probing and irrigation in congenital nasolacrimal duct obstruction. Ophthalmology 1987; 94: 698-705.

13 Robb R. Probing and irrigation for congenital nasolacrimal duct obstruction. Arch Ophthalmol 1986; 104: 378-379.

14 Kushner BJ. The management of naso-lacrimal duct obstruction in children between 18 months and 4 years old. J AAPOS 1998; 2: 57-60. 
15 Honavar S, Prakash V, Rao G. Outcome of probing for congenital nasolacrimal duct obstruction in older children. Am J Ophthalmol 2000; 130: 42-48.

16 Kashkouli MB, Kassaee A, Tabatabaee Z. Initial nasolacrimal duct probing in children under age 5 : cure rate and factors affecting success. J AAPOS 2002; 6 : 360-363.

17 Kashkouli MB, Beigi B, Parvaresh MM, Kassaee A, Tabatabaee $\mathrm{Z}$. Late and very late initial probing for congenital nasolacrimal duct obstruction: what is the cause of failure? Br J Ophthalmol 2003; 87: 1151-1153.

18 MacEwen CJ, Young JDH. The fluorescein disappearance test: an evaluation of its use in infants. J Paediatr Ophthalmol Strabismus 1991; 28: 302-305.

19 MacEwen CJ, Young JDH, Barras CW, Ram B, White PS. The value of nasal endoscopy and probing in the diagnosis and management of children with congenital epiphora. $\mathrm{Br} J$ Ophthalmol 2001; 85: 314-318.

20 Ram B, Barras CW, White PS, MacEwen CJ. The technique of nasendoscopy in the evaluation of nasolacrimal obstruction in children. Rhinology 2000; 38: 83-86.

21 Menard S. Applied logistic regression analysis. In: Quantitative Applications in the Social Sciences, 2nd edn. Series No. 106. Sage Publications: Beverly Hills, CA, p 39.

22 Robb R. Success rates of nasolacrimal duct probing at time intervals after 1 year of age. Ophthalmology 1998; 105: 1307-1310.

23 El-Mansoury J, Calhoun J, Nelson LB, Harley RD. Results of late probing for congenital nasolacrimal duct obstruction Ophthalmology 1986; 93: 1052-1054.
24 Mannor G, Rose G, Frimpong-Ansah K, Ezra E. Factors affecting success of nasolacrimal duct probing for congenital nasolacrimal duct obstruction. Am J Ophthalmol 1999; 127: 616-617.

25 Paul TO, Shepherd R. Congenital nasolacrimal duct obstruction: natural history and the timing of optimal intervention. J Paediatr Ophthalmol Strabismus 1994; 31 362-367.

26 Young JDH, MacEwen CJ, Ogsten S. Congenital nasolacrimal duct obstruction in the second year of life: a multi-centre trial of management. Eye 1996; 10: 185-191.

27 Sener EC, Onerci M. Reappraisal of probing of the congenital obstruction of the nasolacrimal system: is nasendoscopy essential? Int J Paediatr Otorhinolarnygol 2001; 58: $65-68$.

28 Gardiner JA, Forte V, Pashby R, Levin A. The role of nasal endoscopy in repeat paediatric nasolacrimal duct probings. I AAPOS 2001; 5: 148-152.

29 Ingels K, Kestelyn P, Meire F, Ingels G, Van Weissenbruch R. The endoscopic approach for congenital nasolacrimal duct obstruction. Clin Otolaryngol 1997; 22: 96-99.

30 Wesley RE. Inferior turbinate fracture in the treatment of congenital naso-lacrimal duct obstruction and congenital naso-lacrimal duct anomaly. Ophthalmic Surg 1985; 16: 368-371.

31 Havins WE, Wilkins RB. A useful alternative to silicone intubation in congenital nasolacrimal duct obstruction. Ophthalmic Surg 1983; 14: 666-670.

32 Sturrock SM, MacEwen CJ, Young JDH. Long term results after probing for congenital nasolacrimal duct obstruction Br J Ophthalmol 1994; 78: 892-894. 